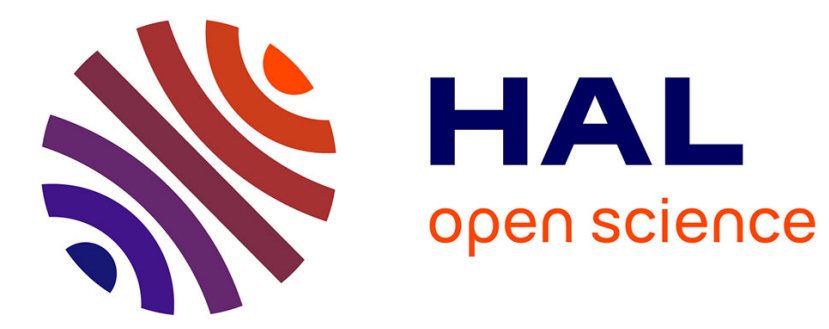

\title{
Kafka à Francfort
}

Alain Vanier

\section{- To cite this version:}

Alain Vanier. Kafka à Francfort. Figures de la psychanalyse, 2007, Kafka, le procès du sujet, 2 (16), pp. 225-238. 10.3917/fp.016.0225 . hal-01524024

\section{HAL Id: hal-01524024 https://hal.science/hal-01524024}

Submitted on 5 Jun 2017

HAL is a multi-disciplinary open access archive for the deposit and dissemination of scientific research documents, whether they are published or not. The documents may come from teaching and research institutions in France or abroad, or from public or private research centers.
L'archive ouverte pluridisciplinaire HAL, est destinée au dépôt et à la diffusion de documents scientifiques de niveau recherche, publiés ou non, émanant des établissements d'enseignement et de recherche français ou étrangers, des laboratoires publics ou privés. 


\section{Kafka à Francfort}

\section{- Alain Vanier •}

La place de l'œuvre de Kafka dans les travaux de l'école de Francfort tient avant tout à l'intérêt que lui portait Walter Benjamin. C'est par lui qu'Adorno a été conduit à fréquenter le texte de Kafka comme en témoigne leur correspondance ; l'article qu'il lui consacre est de tous le plus récent. Sa lecture a accentué un certain versant politique, dont le livre récent de Michael Löwy fournit une illustration probante ${ }^{1}$. Je voudrais plutôt montrer comment les approches de Benjamin surtout, mais aussi d'Adorno, nous aident à lire Kafka et à interroger la façon dont il nous enseigne quelque chose propre à soutenir certaines questions que nous pose aujourd'hui notre pratique de psychanalyste. Donc un Kafka pour les analystes avec Benjamin et Adorno, mais aussi Lacan qui en a fort peu parlé, mais en a parlé tout de même. Ces auteurs rendent riche de résonances l'œuvre de Kafka pour les psychanalystes, en dégageant ce qu'il manifeste et qui nous importe au plus haut point aujourd'hui. Cette lecture sera donc orientée par ce souci et je revendique toutes les infidélités auxquelles il me conduit.

Kafka est né en 1883, Benjamin en 1892 ; Kafka est mort en 1924 et Benjamin en 1940. Ils ne se sont pas rencontrés, bien que cela ait failli se faire en 1916 à Munich, lors d'une lecture publique de La colonie pénitentiaire : Benjamin envisage d'y aller, mais au dernier moment ne peut s'y rendre. La rencontre est manquée, elle n'en sera pas moins réussie sur un autre plan.

La réflexion de Benjamin, dans les textes qu'il consacre à Kafka, s'organise autour de quelques axes principaux, dont certains nous serviront de guides. Le premier texte, Franz Kafka: lors de la construction de la Muraille de Chine, a été lu à la radio de Francfort le 3 juillet $1931^{2}$; le deuxième, Franz Kafka : pour le dixième anniversaire de sa mort ${ }^{3}$, fut publié en décembre 1934 de façon incom-

1. M. Löwy, Franz Kafka rêveur insoumis, Paris, Stock, 2004.

2. W. Benjamin, QEuvres complètes, t. 2, trad. M. de Gandillac, R. Rochlitz, P. Rusch, Paris, Gallimard, Folio, 2000.

3. Ibid. 
plète dans la Jüdische Rundschau ${ }^{4}$ et semble avoir été écrit lors d'un séjour de Benjamin chez Brecht à Swendborg. Brecht a toujours trouvé Benjamin trop mystique. Dans ses discussions avec lui sur cet essai, il soupçonne Kafka de « favoriser le fascisme juif 5 ». Ces deux dates de publication encadrent 1933. Un troisième texte peut être recomposé à partir des multiples références à Kafka dans la correspondance de Benjamin avec Adorno, Brecht, et surtout Gershom Scholem.

Ces échanges concernant Kafka avec Brecht et Adorno d'une part, et Scholem de l'autre, montrent les deux pôles entre lesquels se tisse la lecture de Kafka par Benjamin. Les thèmes de cette oscillation se retrouvent d'une façon manifeste dans les nombreuses réécritures de L'œuvre d'art à l'époque de sa reproduction technique - il en existe au moins cinq versions - avec des modifications sensibles de l'interprétation et des perspectives qu'implique la disparition de l'aura. Doiton, nostalgiques, chercher à la restaurer ? Ou au contraire, doit-on nourrir l'espoir de l'avènement d'un autre monde, d'une autre culture, cette disparition annonçant la fin des illusions d'un certain passé ? Ces interrogations sur une certaine position dans la modernité sont aussi les nôtres.

Comme souvent chez Benjamin, un texte se répète dans l'autre, non sans nuances, et ces variations ont toutes leur importance. Kafka est une sorte de double pour Benjamin, dira Hannah Arendt, à partir duquel il pose le problème de sa relation au judaïsme et à la modernité. Il est pour Benjamin la figure de quelqu'un qui a échoué. Cette remarque se trouve à la fin de cette lettre de 1938 adressée à Scholem ${ }^{6}$. À un certain niveau, elle valide l'observation d'Hannah Arendt, que conforte le parcours de Benjamin avec ses échecs universitaires et littéraires. Mais cet échec, pour l'un comme pour l'autre, manifeste plutôt une « impuissance ontologique » qui, pour Hannah Arendt, caractérise une certaine position juive dans la modernité. De ce point de vue, on peut citer cet échange de Max Brod avec Kafka, que retiendra Benjamin : « Je me rappelle un entretien avec Kafka où nous étions partis de l'Europe actuelle et du déclin de l'humanité. "Nous sommes, disait-il, des pensées nihilistes, des idées de suicide qui naissent dans l'esprit de Dieu." Ce mot me fit aussitôt penser à la conception du monde

4. Panorama ou Revue juive.

5. Voir R. Kahn, «Image de soi, image de l'autre - Walter Benjamin et Marcel Proust », Europe, $\mathrm{n}^{\circ}$ 804, Paris, avril 1996.

6. W. Benjamin, Correspondance, 1910-1940, établie par G. Scholem et T.W. Adorno,

2 vol., trad. G. Petitdemange, Paris, Aubier-Montaigne, 1979. 
des gnostiques, Dieu comme méchant démiurge, dont le monde est la chute originelle. Mais il protesta : "Non, notre monde est simplement un accès de mauvaise humeur de la part de Dieu, un mauvais jour." Je répondis : “Ainsi, en dehors de cette forme sous laquelle notre monde nous apparaît, il y aurait de l'espoir ?” Il sourit : “Oh ! assez d'espoir, une quantité infinie d'espoir - mais pas pour nous ${ }^{7}$." 》

Benjamin s'écarte résolument des commentateurs de son époque. Il s'oppose d'emblée aux lectures théologiques, alors dominantes, mais aussi psychanalytiques de Kafka. Il faudrait dire " psychologisantes » car, avec Adorno, on peut soutenir que Kafka rejoint Freud et « arrache la psychanalyse à la psychologie ». Adorno met en parallèle la psychanalyse freudienne, attentive aux scories du monde phénoménal, et la façon dont Kafka fait de l'art à partir des scories de la réalité. Ainsi, Benjamin propose « une interprétation attentive aux détails, à l'étrangeté des motifs et des images 8 ». Il s'intéresse à tout ce monde des « aides intermédiaires, de ces étranges créatures qui forment un petit monde intermédiaire, à la fois inachevé et quotidien, à la fois consolateurs et ineptes », comme la souris Joséphine, le petit bossu, etc. Ces figures sont les seules à avoir échappé au giron familial, et, pour celles-ci, il y a peut-être de l'espoir. Les autres sont devenues le souci du père de famille comme Odradek ou Gregor Samsa qui se réveille sous forme d'une vermine dans la maison familiale. Miguel Abensour remarque à quel point le petit fascinait Benjamin ${ }^{9}$. La passion du détail de Kafka serait un moyen de lutte contre l'organisation. Ce choix se retrouve chez Adorno - Minima Moralia - et, par conséquent, dans ce qu'il conçoit comme une méthode de lutte : «Kafka n'a pas prêché l'humilité, il a recommandé l'attitude la mieux éprouvée à l'égard du mythe : la ruse. À ses yeux, la plus faible, la plus petite chance pour que le monde ne finisse pas par avoir raison réside dans le fait de lui donner raison ${ }^{10}$.

7. W. Benjamin, « Franz Kafka. Pour le dixième anniversaire de sa mort », dans CEuvres complètes, t. 2, op. cit.

8. R. Rochlitz, préface aux CEuvres complètes de Walter Benjamin, Paris, Gallimard, 2000.

9. M. Abensour, postface à T.W. Adorno (1951), Minima Moralia. Réflexions sur la vie mutilée, trad. É. Kaufholz et J.-R. Ladmiral, Paris, 2003.

10. T. W. Adorno (1955), Prismes. Critique de la culture et société, trad. G. et R. Rochlitz, Paris, Payot, 2003. 
Il faut tenter de comprendre Kafka à partir du centre même de son monde, en ne l'orientant ni vers la philosophie ni vers la religion, mais en posant la question de savoir pourquoi les plafonds sont toujours bas chez Kafka. En effet, ce « qui réclame d'être attrapé au vol, ce sont des choses que nulle oreille n'est destinée à entendre 11 ».

L'œuvre de Kafka est pour Benjamin une œuvre prophétique qu'il faut lire à partir de l'avenir. Aucun décryptage n'est donc possible, car elle est, par définition, indéchiffrable, toute exégèse reste vaine. Ses paraboles sont indéchiffrables pour nous, comme pour leur auteur. «Il n'empêche que les fragments kafkaïens ne s'intègrent pas tout à fait dans les formes de la prose occidentale, et se rapportent à la doctrine comme la Aggadah à la Halakhah. Il ne s'agit pas d'allégories, mais on ne doit pas non plus les prendre à la lettre ; elles sont faites pour pouvoir être citées et racontées en guise d'explications. Possédons-nous la doctrine qu'accompagnent les allégories de Kafka, qu'éclairent les gestes de $\mathrm{K}$. et les mouvements de ses animaux ? Cette doctrine n'est pas donnée ; tout au plus pouvons-nous dire que tel ou tel élément y renvoie de façon allusive - Kafka aurait peut-être dit : la transmet comme vestige. À nos yeux, pourtant, ces éléments peuvent tout aussi bien apparaître comme les précurseurs de la doctrine à venir. En tout état de cause, il s'agit ici de l'organisation de la vie et du travail dans la communauté humaine. Cette question a d'autant plus préoccupé Kafka qu'elle lui apparaissait moins élucidable. Dans son célèbre entretien avec Goethe, à Erfurt, Napoléon avait substitué la politique au destin ; Kafka, variant ce mot, aurait pu définir l'organisation comme destin ${ }^{12}$. " Brecht déjà remarquait : «Kafka n'a eu qu'un problème, celui de l'organisation. Ce qui l'a saisi, c'est l'angoisse devant l'État fourmilière, la façon dont les hommes s'aliènent eux-mêmes par les formes de leur vie commune. Il a prévu certaines formes de cette aliénation, comme les méthodes de la GPU, car on voit avec la Gestapo ce que peut devenir la Tcheka. " Ainsi, pour Benjamin, «chaque jour de sa vie, Kafka était confronté à des conduites indéchiffrables et à des interpellations confuses. Il est possible qu'à l'heure de la mort, il ait voulu rendre à ses contemporains la monnaie de leur pièce ${ }^{13}$. $»$

11. W. Benjamin, Correspondance, 1910-1940, op. cit.

12. W. Benjamin, « Franz Kafka. Pour le dixième anniversaire de sa mort », dans CEuvres complètes, t. 2, op. cit.

13. Ibid. 
Le monde de Kafka, c'est-à-dire le nôtre, est indéchiffrable car il est comme revenu au temps d'avant la révélation. Pour Benjamin, le monde contemporain relève du mythe, voire de l'avant-mythe. Le mythe pour Benjamin renvoie à l'idolâtrie et précède le monothéisme. L'introduction du monothéisme, remarque Lacan, aura modifié les rapports de la vérité et du savoir, là où le mythe donnait à la vérité, en la situant, une place de bouchon. Pour Benjamin, le monde de Kafka est un monde d'avant le mythe, monde préhistorique, archaïque, car le mythe offre déjà une promesse de salut. Mais en même temps, il hésite et remarque qu'il y a chez Kafka trois sources : une source juive, une source chinoise et une source grecque : Ulysse, par exemple, se tient sur le seuil qui sépare le mythe et le conte.

Adorno de même, dans un texte de l'après-guerre, s'élève contre l'annexion philosophique de Kafka par l'existentialisme ${ }^{14}$. Il faut tout prendre à la lettre. Cette œuvre est l'effet des Lumières et de la rationalité dominatrice fondée sur une violence aveugle qui se reproduit elle-même à l'infini, et dont la manifestation la plus moderne est le contrôle bureaucratique. «L'obscurcissement, la décapitation de l'intention parabolique sont des conséquences des Lumières. Plus elles réduisent l'objectivité à l'humain, plus les contours de l'étant pur et simple, qu'elles ne peuvent jamais totalement ramener à la subjectivité et qu'elles ont pourtant vidé de ce qu'il avait de familier, s'étendent devant elles, d'autant plus désespérants et impénétrables. Dans l'esprit des Lumières, Kafka réagit contre le retour à la mythologie. » Kafka est du côté de l'allégorie et non du symbole. Selon Benjamin, Kafka écrit des paraboles ; pour Adorno, ce sont des paraboles dont la clef a été dérobée, et chaque phrase de Kafka nous dit : «Interprètemoi ", et aucune d'entre elles ne tolère l'interprétation. En effet, ce que montre Kafka, c'est qu'aujourd'hui le rapport entre le lecteur et le texte a été perturbé. Chaque phrase nous dit : « Ça m'est familier, mais d'où ? » L'œuvre de Kafka est le règne du « déjà vu ». Si nous étendons ce déjà vu au déjà dit, nous rencontrons la lecture de Benjamin sur le statut de la Loi chez Kafka.

Pour Benjamin, Kafka est, avant tout, l'homme qui ajourne. « Comme les Aggadah du Talmud, les livres de Kafka offrent des récits qui sont toujours laissés en suspens, qui s'attardent dans les descriptions les plus minutieuses, comme s'ils espéraient et, simultanément, redoutaient que le précepte et la formule de

14. T.W. Adorno, "Réflexions sur Kafka », dans Prismes. Critique de la culture et société, op. cit. 
la Halakhah, de la doctrine, puissent les surprendre en cours de route ${ }^{15}$. " Les textes du Talmud et de la Mishna sont traditionnellement distribués entre deux registres. Ce qu'on appelle la Halakhah comprend tous les débats et décisions d'ordre juridique ; la Aggadah est tout le reste, souvent des allégories, de petits récits provenant de la Bible, du folklore ou des légendes, où le sens n'est pas toujours explicitement donné. Parfois, il sert à confirmer tel élément de la loi ou de la jurisprudence, parfois pas. Les textes de Kafka sont à lire comme une création de récits pour ne pas atteindre à l'exégèse législative à laquelle ils devraient aboutir : «Ce qu'il s'agit d'ajourner en permanence, c'est le moment de l'énonciation de la loi. Ce qui se complaît dans ses développements infinis chez Kafka, c'est précisément la peur de la fin. [...] La seule et unique vertu providentielle du fragment chez Kafka est qu'il empêche la loi de jamais s'énoncer comme telle. »

En quelque sorte, Lacan rejoint Benjamin. Il y a peu de références à Kafka dans l'enseignement de Lacan. Son nom propre est cité une première fois dans le séminaire sur "Le Moi », puis en 1962 dans le séminaire sur "L’identification ", ainsi que l'adjectif kafkaïen dans ce même séminaire, et, de nouveau, en 1969. Dans les deux séances, en 1962 et 1969, les mêmes thèmes sont abordés avec le même découpage. Il s'agit à chaque fois de préciser l'articulation du sujet à l'Autre, son moment d'émergence, la fonction du trait unaire, et son rapport au désir, à la demande et à la jouissance de cet Autre. La question posée est celle du savoir et de la subjectivité de l'Autre. Ces références sont situées dans le champ de la névrose avec, à chaque fois, un développement sur la clinique des névroses, et, plus particulièrement, de l'obsessionnel. Un hasard ? D'autant qu'en 1969, ce retour sous forme adjectivée semble être venu de façon non calculée, non préparée au moment où Lacan reprend et réarticule ce qu'il avait évoqué sept ans plus tôt. Remarquons, brièvement, qu'en 1962 Lacan s'intéresse à la question de l'eksistence du sujet dans son rapport à la construction de l'objet, c'est-à-dire à la constitution comme telle du «sujet dans le désir », soit le chemin qui va de la frustration marquée par la dominance de l'Autre à la castration en jeu dans l'œdipe. Lacan rappelle qu'il existe un « nœud interne » qui est « un rapport entre une demande qui prend une valeur si privilégiée qu'elle devient le commandement absolu, la loi, et un désir, lequel est le désir de l'Autre dont il s'agit dans l'œdipe. Cette demande s'articule ainsi : tu ne désireras pas celle qui a été mon désir. » Ainsi, tout désir inclut en lui ce trou interne spécifié dans ce

15. W. Benjamin, « Franz Kafka: lors de la construction de la Muraille de Chine », dans CEuvres complètes, t. 2, op. cit.. 
rapport à la Loi originelle qui s'exprime, qui passe par une demande, « demande de l'Autre » où le névrosé fonde son désir. Cela signifie que toute demande du sujet suppose un déjà là, un déjà dit de la loi. L’ajournement kafkaïen implique une loi déjà là, sinon aucune raison d'ajourner. La pression à laquelle nous soumet le névrosé dans l'analyse est fondamentalement l'exigence d'une réponse attendue, réponse sur «son désir et sur sa satisfaction ». Cette demande : «Qu'est-ce que je veux ? », est à entendre sur le fond de la première demande de l'Autre, demande sidérante qui le décomplète : que veux-tu ? Lacan insiste sur le balancement entre la possibilité, la puissance d'être et le rien comme réponse, entre le «qu'est-ce que je veux ? » et la réponse qui va du «peut-être rien », comme réponse éventuelle au niveau du message, en référence au « rien peut-être ? » comme question, « rien peut être », c'est-à-dire que rien ne soit déterminé de cette question sur le désir. Ce balancement oscille entre le « rien n'est sûr », le possible de ne pas pouvoir conclure « si ce n'est par le recours à l'antériorité infinie du procès kafkaïen », c'est-à-dire ce recours au temps d'avant, comme tentative pour conclure. De cet insuccès nécessaire témoignent les inachèvements multiples chez Kafka, aussi bien de ses livres que de son lien matrimonial aux femmes. Il se maintient ainsi avant ce point décisif où l'Autre répond « rien », que « rien n'est sûr ». Il manifeste ainsi ce défaut de savoir dans l'Autre, voire sa volonté de ne pas savoir. Or, c'est cette défaillance de l'Autre comme garantie du sûr qui est redoutée chez Kafka, et c'est ce que montrera Benjamin.

Dans cette même séance, Lacan, à propos de ce nœud de la topologie du tore, dira que « l'homme est un animal de tore » et non de sphère, comme la philosophie l'a jusqu'à présent soutenu, mais aussi tout un courant de la psychanalyse. L'homme est un animal de tore, « un animal de terrier », ajoute-t-il, en évoquant le texte de Kafka. À ce propos, il évoque le mode d'engagement du névrosé dans un certain rapport à l'Autre, que la nouvelle de Kafka manifeste par l'importance de ce bruit dans le terrier comme signe de l'Autre : «Que veut-il ? »

Sept ans plus tard, Lacan utilise à nouveau cet adjectif «kafkaïen », quand il aborde les mêmes questions : Est-ce que l'Autre sait ? Est-ce qu'il est un sujet ? Le sujet supposé savoir, précise-t-il à cette occasion, ne signifie qu'une chose : que l'Autre est le lieu de l'inconscient, qu'il sait, mais qu'il n'est pas sujet. L'objet se présente comme caché, et cette dimension du caché instaure la réalité du désir, car elle infère qu'il y a un objet qui existe. Cet objet caché est la source de la foi en Dieu. 
"Comment penser, au sens de Kafka, la projection du Jugement dernier dans le cours du monde ? » écrit Benjamin à Scholem, quand il rédige son étude. « Cette projection transforme-t-elle le juge en accusé ? La procédure en châtiment ? Est-ce voué à dresser ou à enterrer la loi ? " Et commentant ainsi son étude, il ajoute : « J'ai tenté de montrer comment c'est au revers de ce néant, dans sa doublure si j'ose dire, que Kafka a cherché à effleurer du doigt la rédemption. "

De là, Benjamin pose la question du Père, des pères, de ces figures des puissants qui sont autant d'images du Père chez Kafka. « Ces puissants nous apparaissent dans un mouvement lent et continu - déclinant ou ascendant. Mais ils ne sont nulle part si terribles que lorsqu'ils surgissent de la plus profonde dégradation : d'entre les pères. Le père hébété et sénile, que le fils vient de coucher doucement en lui disant : "Sois tranquille, tu es bien couvert", ce père s'écrie : “"Non !" [...] avant même que la réponse fût finie, et il rejeta la couverture avec une telle force qu'elle se déploya un instant tout entière dans son vol, puis il se dressa debout sur son lit. Il s'appuyait seulement d'une main au plafond : “Tu voulais me couvrir, je le sais, petit vaurien, mais je ne suis pas encore couvert; ce sont peut-être mes dernières forces, mais c'est assez pour toi, c'est même trop ! [...] Mais heureusement personne n'a besoin d'apprendre à un père à voir dans le cœur de son fils” [...]. Il se tenait debout, parfaitement libre, et lançait des coups de pied. Il rayonnait d'intelligence [...]. “Tu sais donc maintenant ce qu'il y a eu en dehors de toi ! Jusqu'ici tu ne connaissais rien d'autre que toi-même! Tu étais au fond un enfant innocent, mais plus au fond encore un être diabolique !“ En rejetant le poids de la couverture, le père rejette en même temps une charge universelle » écrit Benjamin, et, commentant ce passage du Verdict, il ajoute : «Ce sont les âges du monde qu'il doit remuer pour faire de l'immémoriale relation père-fils une relation vivante et riche de conséquences. Mais de quelles conséquences ! Il condamne le fils à la mort par noyade. Le père est celui qui punit. La culpabilité l'attire comme elle attire les fonctionnaires de justice. Tout indique que, pour Kafka, le monde des fonctionnaires et celui des pères se confondent. La ressemblance n'est pas à leur honneur. Elle est faite d'hébétude, de dégradations et de crasse. L'uniforme du père est constellé de taches, son linge de corps est douteux ${ }^{16}$. » Le fonctionnaire est donc un parasite qui se nour-

16. W. Benjamin, « Franz Kafka. Pour le dixième anniversaire de sa mort », dans CEuvres complètes, t. 2, op. cit. 
rit des forces d'humanité et de raison. «C'est de la même façon, dans les étranges familles de Kafka, que le père se nourrit du fils, pèse sur lui comme un monstrueux parasite. Le père ne ronge pas seulement la force du fils, il ronge son droit d'exister. Le père, celui qui punit, est aussi celui qui accuse. La faute dont il accuse le fils semble une sorte de péché originel. De fait, la définition que Kafka donne de celui-ci ne s'applique à personne mieux qu'au fils : “Le péché originel, cette vieille injustice que l'homme a commise, consiste dans le reproche que l'homme fait et auquel il ne renonce pas, à savoir qu'une injustice a été commise à son égard, qu'il a été victime d'un péché originel." Mais qui est accusé de ce péché originel - le péché d'avoir engendré un héritier - sinon le père par le fils ? Le pécheur serait donc le fils. Du principe posé par Kafka, on ne peut pourtant conclure que l'accusation est un péché parce qu'elle est fausse. Nulle part, il ne dit qu'elle est portée à tort. " Le père renvoie donc sans cesse le fils à une sorte de péché originel, son image structure un monde où le pouvoir divin est devenu inintelligible ${ }^{17}$. Le père d'aujourd'hui n'endosse plus la culpabilité au nom du Père. Dès lors, le sujet n'a plus affaire à son désir, mais à l'horreur de sa jouissance. Car ce qui le garantissait contre, ce qui la limitait, est aussi ce à quoi le sujet se refuse. N'est-ce pas là aussi une manière de maintenir vivant ce qui du père est toujours déjà mort, et d'ajourner ainsi son propre désir ? Le questionnement inlassable sur le désir du père n'est-il pas un mode pour le faire exister comme défense ? En effet, souligne Adorno, « la suspension des règles de la société patriarcale en révèle le secret, celui d'une répression directe, barbare ». Faut-il alors penser avec Scholem que «Benjamin découvre dans le monde de Kafka l'inversion négative (negativer Umschlag) de catégories juives. Plus de doctrine positive. Seule subsiste une promesse strictement utopique qu'on ne savait encore formuler. Benjamin savait que l'on trouve dans Kafka la "theologia negativa“ d'un judaïsme qui a perdu le sens positif de la Révélation mais qui n'a rien perdu de son intensité. » On n'est pas loin de Marthe Robert qui soulignait que « là où les Juifs assimilés n'ont rien de plus pressé que de s'affranchir des contraintes rituelles incompatibles avec leur désir de se fondre dans leur milieu, Kafka regrette les contraintes qu'il n'a pas reçues de son éducation et dont par conséquent, fût-il tenté d'en faire l'essai, il ne saura même pas très bien comment, pourquoi, jusqu'à quel point il devra s'y plier. C'est que pour lui le commandement n'est nullement aboli par la disparition du commandant qui l'énonçait jadis, il survit en quelque sorte à sa propre nécessité ; et non seulement

17. Voir P. Bouretz, Témoins du futur, Paris, Gallimard, 2003. 
il reste seul à parler, mais, s'étant totalement émancipé de l'ordre divin dont il était l'agent dans les "vieux temps formidables", il devient plus contraignant que jamais, et si tyrannique, que ses exigences ne connaissent plus ni mesure ni limites ${ }^{18}$. " Mais Benjamin va au-delà.

Cet ajournement permanent a comme corollaire la peur, la peur de la fin. Ce n'est pas l'angoisse, malgré les lectures et ce que l'on sait ou suppose de connexions avec Kierkegaard. Cette peur, souligne Benjamin, tient au corps dans tous les sens du terme. «De quoi avons-nous peur? De notre corps », pouvait dire Lacan. Car « l'homme d'aujourd'hui vit dans son corps comme K. dans le village au pied du château : il est un étranger, un exilé, qui ne sait rien des lois qui unissent ce corps, à des ordres supérieurs et plus vastes ». En effet, faute d'une médiation assurée jusque-là par la fonction du père, et maintenue ici mais à quel prix, le joint entre le corps et le langage devient problématique. Le problème du névrosé est d'avoir un père qui parle, qui désire et jouit, père qu'il ne se résout pas à réduire à la fonction de passeur de la Loi ou du langage, de celui qui prend en charge la jouissance maternelle. Le père du névrosé parle comme un imposteur à la place du langage. Ce père se constitue ainsi comme symptôme du névrosé. Or, Kafka n'est pas sans le savoir, mais « il renoncera à la vérité pour maintenir la tradition ». Kafka manifeste au plus haut point l'équivalence du père et du sinthome en faisant du sien un symptôme ; pour que cela puisse apparaître, il aura fallu le moment historique où il s'est trouvé. Il le manifeste, car faute de pouvoir complètement le nouer, il reste dans l'inhibition et l'angoisse. Il voudra soutenir le père et virer la jouissance à son crédit. Il est sur la même frontière que Joyce pour Lacan, mais il est juste de l'autre côté. Cela se manifeste dans son écriture où la lettre, l'éclatement du signifiant ne se produit pas ; la langue n'est pas attaquée. On n'y sent pas la jouissance de celui qui écrit quand on le lit, en témoigne la sympathie qu'il suscite chez le lecteur ; au niveau du régime de sa jouissance, il n'y a aucun rapport qui passe par la lettre. L'évolution de son style va dans le sens de cet apurement, bien qu'il soit, comme Joyce, entre plusieurs langues. Il n'est pas hors sens ; au contraire, il est même à ce point initial et radical de l'émergence du sens, c'est-à-dire au point initial de l'énigme. L'énigme que Lacan écrit $E_{e}$ et dont il dit qu'elle est affaire d'énonciation : «pourquoi diable un tel énoncé a-t-il été prononcé ? » Si Joyce est l'écrivain par excellence de l'énigme, à comprendre comme conséquence d'un « raboutage si 
mal fait que c'est un ego de fonction énigmatique », il est évident que Kafka ne se tient pas sur le même bord. Kafka a-t-il voulu se faire un nom ? Pour lui, ce nom, écrasant, n'a ni besoin d'être illustré ou soutenu pour qu'il subsiste. N'a-t-il pas, dans ce rapport à ce nom, manifesté une ambiguïté, une ambivalence dont témoigne l'aventure de son testament d'écrivain ?

Kafka se confronte au pouvoir mortel de l'écriture, d'où son inhibition et l'alternance avec la correspondance dans un mouvement d'appel à l'Autre. La dimension mortelle du signifiant qui est de structure ne lui échappe pas. « L'artiste attendit que K. se fût calmé, puis, ne voyant pas d'échappatoire, se décida à continuer son travail. Le premier trait qu'il inscrivit fut une délivrance pour $\mathrm{K}$., mais l'artiste ne réussit visiblement à l'achever qu'avec la plus grand répugnance ; l'écriture n'était d'ailleurs plus aussi belle, elle semblait surtout manquer d'or, le trait était pâle et incertain mais la lettre fut très grande. C'était un J, il allait être fini quand l'artiste frappa furieusement du pied dans le tertre ; la terre en vola tout autour. K. comprit enfin le graveur ; il n'était plus temps de le retenir ; il creusait déjà de tous ses doigts dans la terre qui n'offrit presque aucune résistance ; tout semblait être prêt ; la mince croûte de terre n'était là que pour l'illusion ; un grand trou aux parois à pic s'ouvrait immédiatement au-dessous, dans lequel K. s'enfonça, renversé sur le dos par un léger courant. Or, tandis qu'il plongeait au cœur de cet abîme insondable, la nuque encore redressée, son nom se dessina là-haut comme un éclair avec d'immenses arabesques sur la pierre. Ravi de ce spectacle, il se réveilla ${ }^{19}$. " Et Adorno commente : "Seul le nom révélé par la mort naturelle, non l'âme vivante, répond de la part immortelle. " Ainsi la lettre apparaît dans le rêve, mais il n'y a nulle part d'équivalent du Finnegans Wake de Joyce et de ce lien entre l'écriture et la jouissance. C'est une autre modalité de ce lien qu'il faut évoquer.

Puisque le joint entre corps et langage devient problématique, c'est la peur qui réunit toutes les créatures chez Kafka, elles sont toutes « secrètement solidaires dans un seul et unique sentiment : la peur. Une peur qui n'est pas une réaction, mais un organe. Et l'on peut aisément déterminer pour quel ordre de phénomènes cet organe possède à tout moment la sensibilité la plus fine et la plus infaillible. Mais avant que son objet ne devienne visible, c'est son ambivalence remarquable qui doit nous donner à réfléchir. Car cette peur - pensons à

19. F. Kafka, « Le procès », trad. A. Vialatte, dans CEuvres complètes, t. I, Paris, Gallimard, «La Pléiade », 1976. 
l'image du miroir qui a été utilisée au commencement - est en même temps et dans les mêmes proportions peur d'un passé immémorial et peur d'un avenir imminent. En un mot, elle est la peur d'une faute inconnue et de son expiation, dont le seul bienfait est de révéler la faute ${ }^{20}$. " Ainsi, faute d'un organe plus ou moins adéquat pour assurer la jonction du corps et du langage et fonder un rapport au monde, la peur fonctionne comme lien social. « En effet, l'homme d'aujourd'hui vit dans son corps comme K. dans le village au pied du château ; il lui échappe, il lui est hostile. Il peut arriver que l'homme un matin se réveille et se trouve transformé en vermine. Le pays étranger - son pays étranger - s'est emparé de lui. » Ce village est celui d'une légende talmudique, c'est l'air «qui souffle chez Kafka, et c'est pourquoi il n'a pas été tenté de fonder une religion 21 ». Adorno aussi souligne comment les personnages dans l'œuvre de Kafka sont toujours sur le bord d'une « régression quasi biologique » où « les hommes s'aperçoivent que loin d'être un moi, ils sont eux-mêmes des choses ». Cen'est pas la construction d'un ego comme correcteur du « rapport manquant » qui est en jeu dans l'écriture de Kafka, mais plutôt de toucher ce point d'objectivation de son être, qui tient à une autre dimension du corps. À quoi fait écho la remarque de Benjamin, à savoir qu'une « chose est sûre : parmi toutes les créatures de Kafka, ce sont les bêtes qui réfléchissent le plus. La peur est dans leur pensée ce que la corruption est dans le droit. Elle gâche le déroulement de l'action, et elle est pourtant en celle-ci le seul élément qui recèle de l'espoir. Mais puisque nous habitons notre corps - notre propre corps - comme le pays étranger le plus oublié, on comprend que cette toux qui éclatait au fond de lui-même, Kafka l'ait appelée la "bête". Elle était le poste le plus avancé de la grande horde. »Kafka a « inlassablement épié dans les bêtes la trace de ce qui a été oublié 22 ». Ajoutons parmi les bêtes notre ancêtre, dont la bestialité a été, n'a pas, ou aurait dû être sacrifiée, en effet, « le monde des ancêtres s'étend pour Kafka à l'infini, et, comme les arbres totémiques chez les primitifs, plonge ses racines dans le règne animal 23. » Dans le séminaire « D'un Autre à l'autre », où émerge pour une dernière fois l'adjectif kafkaïen, Lacan évoque le cheval de

20. W. Benjamin, « Franz Kafka : lors de la construction de la Muraille de Chine », dans CEuvres complètes, t. 2, op. cit.

21. W. Benjamin, «Franz Kafka. Pour le dixième anniversaire de sa mort », dans CEuvres complètes, t. 2, op. cit.

22. Ibid. À Scholem, il écrit : «Être animal pour lui signifie sans doute simplement avoir renoncé, par une sorte de pudeur, à la figure et à la sagesse humaines. »

23. Ibid. 
Troie, cet animal, qu'il suppose non pas plein de guerriers mais vide, vide de ce vide de l'Autre où est appelée cette assemblée humaine, un cheval qui ne se viderait pas de ses guerriers pour les répandre dans Troie, mais « qui en absorbe de plus en plus dans son ventre».

À l'occasion de la parution du Kafka de Max Brod, Benjamin écrit, en 1938, à Scholem pour préciser encore son approche de Kafka, et l'évocation de ce texte majeur nous permettra de conclure : «L'œuvre de Kafka est une ellipse dont les foyers, très éloignés l'un de l'autre, sont définis, l'un, par l'expérience mystique (qui est avant tout l'expérience de la tradition), et l'autre, par l'expérience de l'homme de la grande ville moderne. » L'homme de la grande ville moderne est d'un côté le « citoyen moderne qui se sait livré à un appareil bureaucratique impénétrable dont la fonction est contrôlée par des instances qui restent floues même à ses organes d'exécution, a fortiori pour ceux qu'il manipule ». Cette position singulière évoque aussi bien cette individuation problématique (Adorno), que cette séparation sans espoir d'avec le giron maternel (Benjamin), au sens où Lacan a pu dire qu'aujourd'hui « chaque individu est réellement un prolétaire, c'est-à-dire n'a nul discours de quoi faire lien social, autrement dit semblant »; et la solitude de Kafka, cet irrémédiable, manifeste le symptôme social de l'individu moderne. Le succès de l'adjectif «kafkaïen », qui existe aujourd'hui dans pratiquement toutes les langues, comme le remarquent George Steiner et Michael Löwy, manifeste son lien à la modernité. Mais loin de n’être que victime, il témoigne d'une façon inédite du prix que le sujet moderne paie pour soutenir la structure dans « notre civilisation axée sur la névrose ». Avec lui aussi, mais de l'autre côté d'une certaine limite de la structure, peut se poser la question de savoir « à partir de quand est-on fou? ».

Kafka, souligne Benjamin, est aussi « tout autant le contemporain des physiciens d'aujourd'hui ». Il rapporte le passage suivant du physicien Eddington où « on croit entendre Kafka ». « Je suis sur le pas de ma porte avec l'idée d'entrer dans ma chambre. C'est là une entreprise compliquée. D'abord, je dois lutter contre l'atmosphère qui pèse sur chaque centimètre carré de mon corps avec une force de 1 kilo. Je dois ensuite essayer d'atterrir sur une planche qui vole autour du Soleil à la vitesse de 30 kilomètres par seconde ; une fraction de seconde de retard et la planche est à des milliers de distance. Et il faut réaliser cette prouesse au moment même où je suis suspendu à une planète sphérique, la tête vers le dehors, plongé dans l'espace et un vent de terre souffle on ne sait à quelle vitesse par tous les pores de mon corps [...]. » Donc, si l'on dit, comme je l'ai fait, que les expériences de cette sorte se rapportaient chez Kafka, au prix d'une tension 
violente à ses expériences mystiques, on ne dit qu'une demi-vérité. Ce qu'il y a de fou dans Kafka, de proprement fou au sens précis du terme, c'est que cet univers d'expérience, de tous le plus récent, lui ait été convoyé précisément par la tradition mystique. Cela bien entendu n'a pas été possible sans qu'interviennent des événements dévastateurs au sein de cette tradition même [...]. D'où qu'on le prenne, le problème est que, de toute évidence, il fallait faire appel à rien de moins qu'aux forces de cette tradition, dès lors qu'un individu (qui s'appelait Franz Kafka) devait affronter la réalité qui se dessine comme étant la nôtre, à un plan théorique dans la physique moderne par exemple, ou, au plan pratique, dans la technique de la guerre. Je veux dire que cette réalité n'est pratiquement plus perceptible à l'individu et que le monde de Kafka, souvent si serein et traversé par des anges, est l'exact complément de son époque qui s'apprête à supprimer par masses entières les habitants de cette planète. L'expérience qui correspond à celle de Kafka individu privé pourrait bien n'être acquise par les grandes masses qu'à l'heure de leur propre suppression. »

Si l'histoire est toujours l'histoire des vainqueurs, l'œuvre de Kafka ne peutelle se lire comme une tentative d'écrire l'histoire des vaincus dans ce monde de déportation de corps, devenus chair à canon, où il n'y a plus, sauf illusoirement, ni vainqueur, ni rédemption. Ainsi Kafka est bien la figure de quelqu'un qui a échoué, celle de l'échec fondamental du sujet moderne. Il perçoit le temps qui vient « sans percevoir ce qu'est l'aujourd'hui », car « l'unique racine de son expérience est la tradition à laquelle [il] s'est livré ; aucune sorte de clairvoyance des lointains, pas davantage des «dons de visionnaire ». Kafka était aux écoutes de la tradition, et qui écoute tendu de toutes ses forces, ne voit pas. Il existe un espoir infini, simplement pas pour nous 24 .

\section{RÉSUMÉ}

Une lecture de Kafka avec Benjamin, Adorno et Lacan.

\section{MotS-CLEFS}

F. Kafka, W. Benjamin, T.W. Adorno, Père, Autre, Loi, tradition.

\section{SUMMARY}

An approach to Kafka with Benjamin, Adorno and Lacan.

\section{KeY-WORDS}

F. Kafka, W. Benjamin T.W. Adorno, Father, Other, Law, tradition.

24. W. Benjamin, Correspondance, 1910-1940, op. cit. 